\title{
ROSETTA'S JOURNEY TO COMET CHURYUMOV-GERASIMENKO
}

\section{AN ENCOUNTER TO EXPLORE THE BEGINNING OF THE SOLAR SYSTEM}

„ H. Balsiger ${ }^{1}$ and G. Schwehm ${ }^{2}$ - DOI: 10.1051/epn/2015401

- ${ }^{1}$ Physikalisches Institut University of Bern, Bern $-{ }^{2}$ European Space Agency (retired)

On 6 August 2014, finally the big day had come: ESA's Rosetta mission went into an orbit around comet 67P/ Churyumov-Gerasimenko to start its actual science mission. More than ten years after the launch on 2 March 2004 and more than 6 billion $\mathbf{k m}$ cruise, the science phase, for which a large number of scientists had worked since the mid-nineties of the last century, did start.

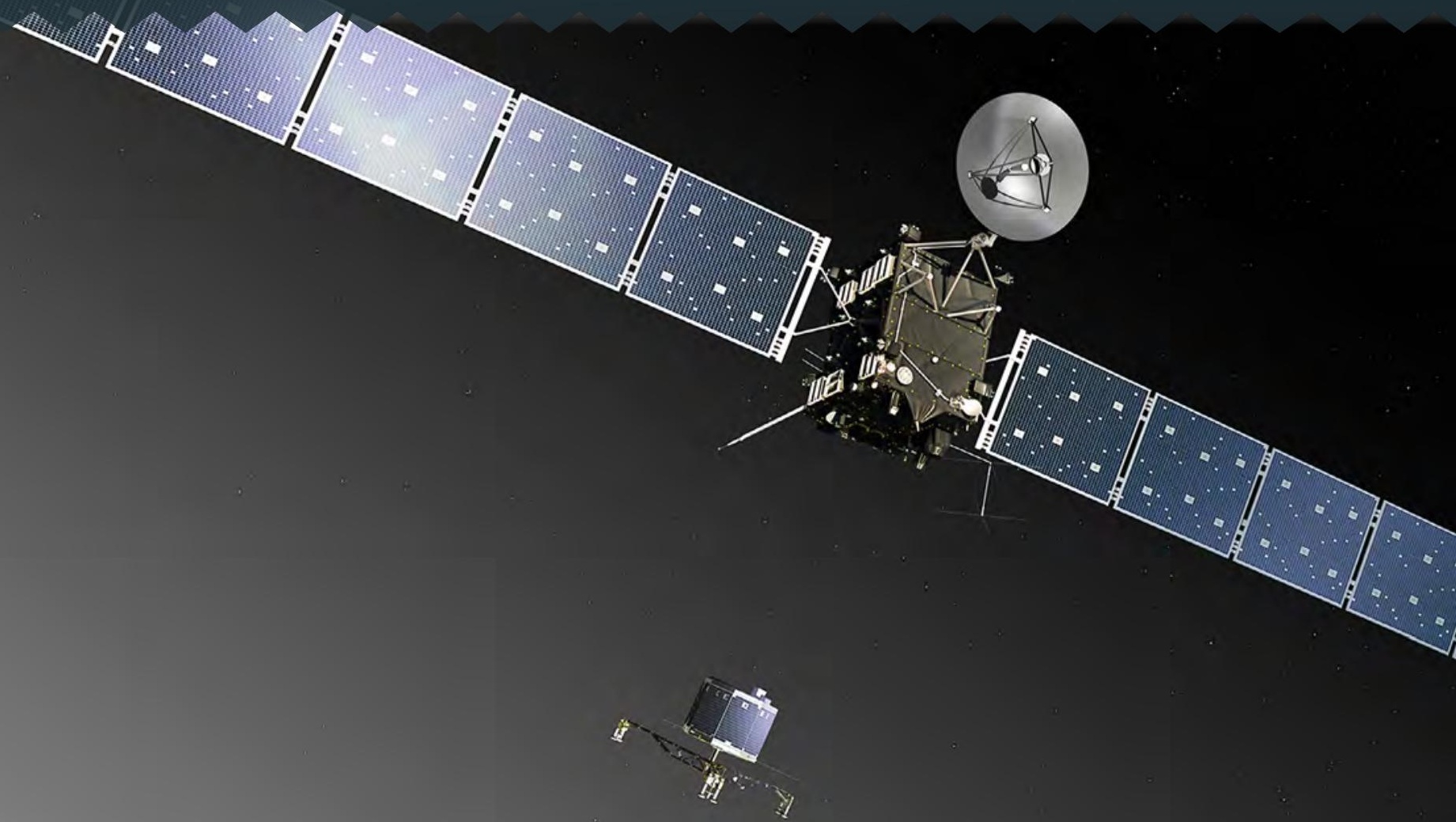




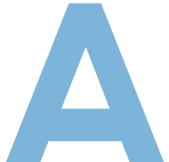

ctually, the preparation work for the mission had already started in 1985, originally perceived as a Comet-Nucleus Sample-Return mission, the fourth cornerstone in ESA's new long- term programme Horizon 2000 [1] jointly with NASA.

However, in the aftermath of the explosion of the shuttle Challenger on 28 January, NASA had to restructure their programme and Europe had to define a mission that could be done by ESA alone. Rosetta re-emerged as a comet rendez-vous mission with asteroid fly-bys: 'If we cannot bring back material out of a comet nucleus to our laboratories on Earth, we will bring the laboratory to the comet' became the new slogan.

In 2002 spacecraft and payload were ready (Fig. 1) and tested for the launch in early 2003 with the Ariane 5 from Kourou to comet 46P/Wirtanen. The spacecraft was already in Kourou and fueled, when the improved version of the Ariane 5 exploded on her maiden flight, shortly after lift off just before Christmas 2002. As a consequence the launcher was grounded until the problem had been identified and resolved.

In a very short time an alternative mission scenario was found: the next opportunity with an Ariane 5 was the mission to comet 67P/Churyumov-Gerasimenko with a launch in March 2004.

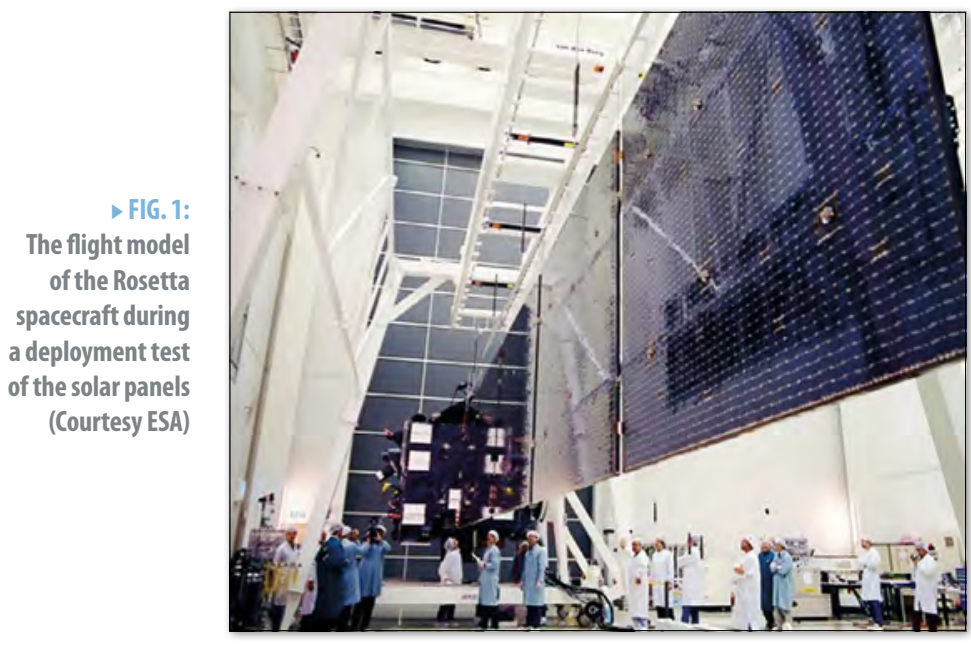

The Mission

On 2 March 2004 the journey to the comet started. It took more than ten years, with three Earth gravity assists and one at Mars to gain sufficient orbital energy, to get Rosetta to its rendez-vous with 67P/ Churyumov-Gerasimenko.

On its long journey the mission returned the first exciting science from the two asteroid flybys at 2867 Steins in September 2008 and at 21 Lutetia in July 2010, respectively.

Mid 2011 Rosetta was put into a 30 months hibernation as, despite the huge solar arrays, at a distance beyond 4 a.u. from the Sun, not enough energy could be generated to operate the spacecraft under all conditions.
On 20 January 2014 Rosetta woke up by its internal wake-up call and the most important phase of its mission was about to begin. On 6 August 2014 it entered the orbit around the nucleus at a distance of $100 \mathrm{~km}$. An extensive science and reconnaissance phase started with the initial prime objective to select a landing site for its surface science laboratory Philae.

The first soft landing on a comet nucleus occurred on 12 November, 2014. At 8:35 UTC the Lander was released with a relative velocity $\Delta v=0.1876 \mathrm{~m} / \mathrm{s}$ about $20.5 \mathrm{~km}$ from the nucleus surface. After 6:59:04 hours of ballistic descent Philae landed on the comet at 15:34:03.97 UTC with a touchdown speed relative to the surface of about 1 $\mathrm{m} / \mathrm{s}$. Since the anchor harpoons did not fire upon touchdown and the hold-down thrust of the cold-gas system did not work either, the lander bounced several times until it came to rest after 1:57 hours of ballistic flight at its final landing site.

\section{Science goals}

Already late in the $17^{\text {th }}$ century Edmond Halley and Isaac Newton had independently proposed that comets may have played an important role in the development of planets [2]. Whereas Halley thought that a collision with a comet could have caused a biblical flood on Earth, Newton proposed that the vapours from comet tails could have been collected by planetary atmospheres, thereby delivering the water necessary for terrestrial life. These were quite bold suggestions if one considers that, at the time, nobody knew the chemical composition or the structure of comets. Later, after detection of carbon-bearing radicals in the spectra of comets, it was speculated that their parent molecules could have been the source of the pre-biotical chemistry that ultimately resulted in the development of life on Earth.

The two open questions, origin of water and life on Earth, are still drivers for comet science together with the question how the solar system has been formed and developed. That comets play an important role in this basic question is taken for granted. The fact that they represent the most primitive material found in the solar system and hence are witnesses of the very early history has already been demonstrated by composition measurements during the Giotto mission [3]. The role of comets in the formation history of the solar system has been the subject of numerous models and speculations. Of the two families, the Comets in the Oort Cloud and those in the Kuiper Belt, at least the latter formed beyond the orbit of Neptune at very low temperatures. They are considered to originate from the family of icy planetesimals that have contributed to the volatile inventory of the planets, including the terrestrial planets. Impacts of comets must have brought some water and other volatiles to the Earth and the inner planets. However the magnitude of their contribution is debated. 
The Rosetta mission [4] to 67P/Churyumov-Gerasimenko, a "Jupiter-family comet" (originating in the Kuiper belt) has the prime goal to solve as many of the basic open questions as possible by accompanying the comet from aphel through perihel and monitoring its development and activity. For this purpose a suite of twelve sophisticated instruments covering all conceivable aspects was selected for Rosetta's orbiter. Ten more instruments on the lander Philae are characterizing structure and composition of the surface of the nucleus.

\section{First results}

The first results of the measurments in October/November 2014 are already very exciting and in part also quite surprising. Due to the fact that the comet's activity was substantial and the search of the Philae landing site was performed in a bound orbit of $10 \mathrm{~km}$ altitude, a first science campaign could be carried out, which has led to a Special Issue of Science with unprecedented details of a comet nucleus [5]. We will here give a summary of these first results.

\section{Shape, Density and Surface Structure}

Already during approach it became clear that this comet has a funny shape and a wealth of surface structure elements which are not easily explained, considering its low gravity forces. Its duck-like shape (Fig. 2) raises the question whether the two lobes represent a contact-binary or a single body where a gap has evolved via mass loss. The duck rotates in 12 hours around the axis shown in Fig. 2 , and this axis appears to be stable $\left(<0.3^{\circ}\right)$. The total volume is currently estimated to be $21.4 \pm 2.0 \mathrm{~km}^{3}$, whereby the uncertainty reflects the fact that the entire nucleus has not yet been seen). This leads to a mean density of $470 \pm$

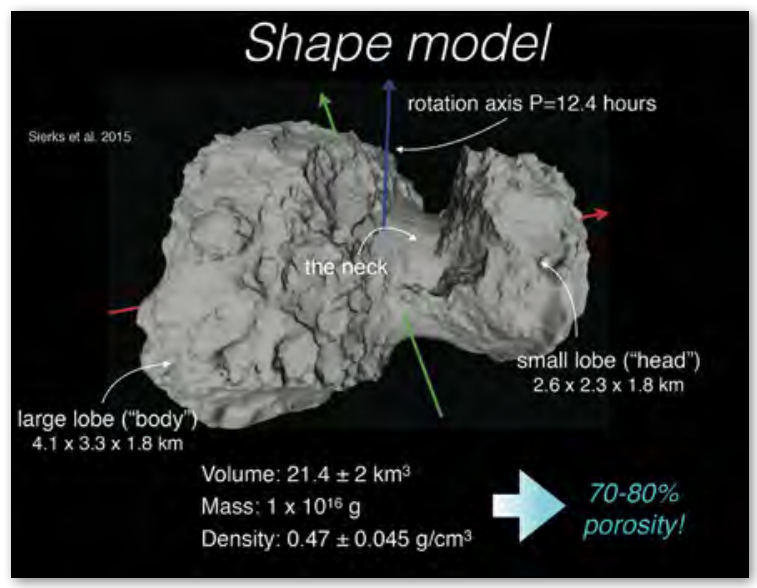

4 FIG. 2 :

Stereophotogrammetric shape model of the nucleus, supplemented with vital statistics (5) (Courtesy ESA)

$45 \mathrm{~kg} / \mathrm{m}^{3}$. Assuming that this density is homogeneous, a high porosity of $70-80 \%$ is implied.

High resolution pictures $(0.8 \mathrm{~m} / \mathrm{Pixel})$ reveal an amazing variety of surface textures. Cliffs as high as $900 \mathrm{~m}$, several hundred meters long fractures, circular pits and boulders up to several meters are just some of the impressive features. Their origin can result from the thermal shock by the very large variation in temperatures $(150 \mathrm{~K})$ seen by comet nuclei or collapse of internal reservoirs of volatiles. But only the development during approach of the Sun will give more evidence on the mechanisms in place.

\section{Composition of nucleus and coma}

Remote-sensing instruments have measured the progression of the $\mathrm{H}_{2} \mathrm{O}$ production rate. It has developed from $0.3 \mathrm{~kg} / \mathrm{s}$ in June 2014 to $1.2 \mathrm{~kg} / \mathrm{s}$ in late August. With an albedo of 0.060 , the comet is very dark. Broad absorption features taken in radiation with low micrometer wavelengths that are present across the entire illuminated surface, are compatible with

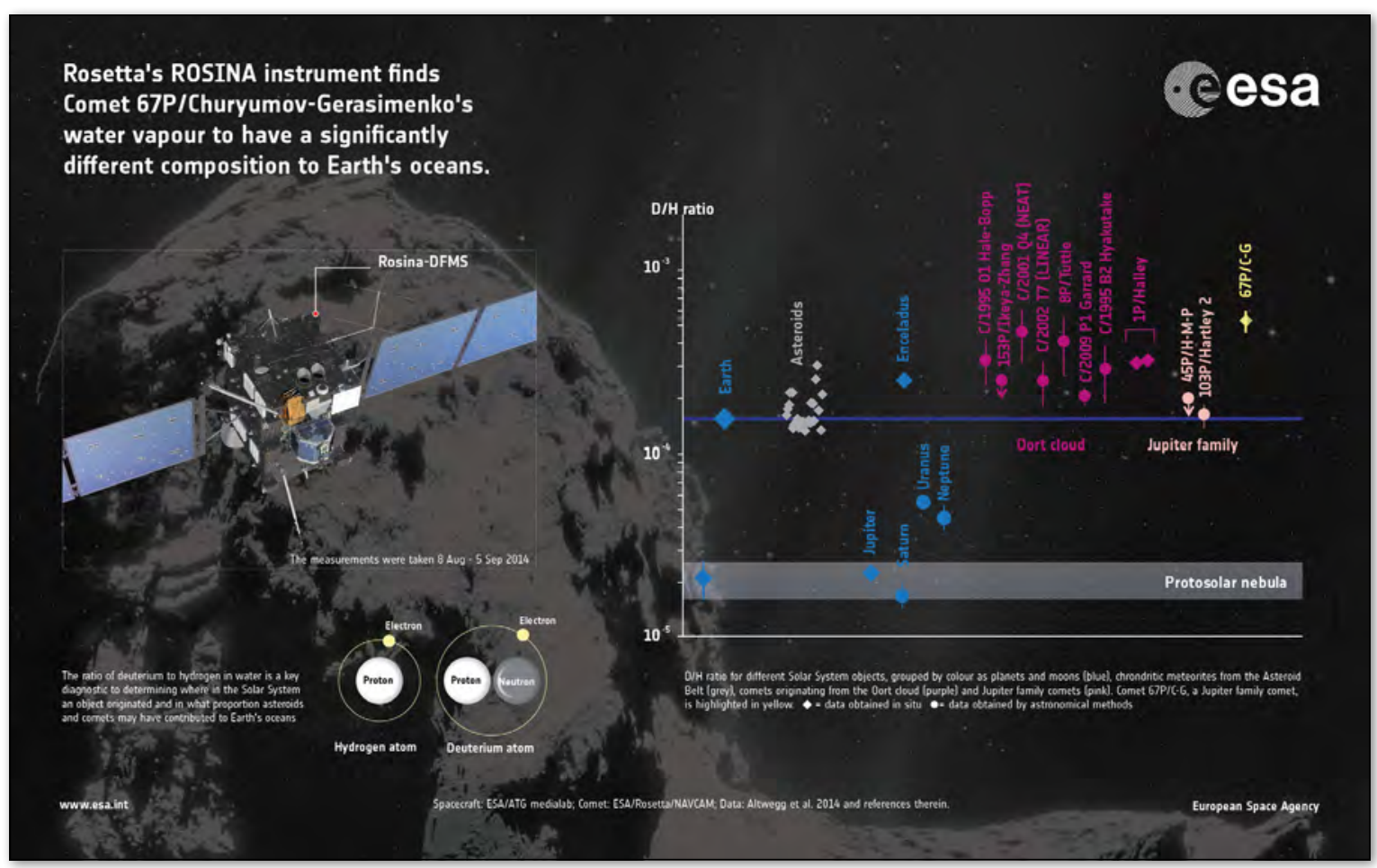

4 FIG. 3:

The isotopic ratio between deuterium and hydrogen (D/H) measured after the rendez-vous with comet 67P/ ChuryumovGerasimenko in comparison with that of other bodies belonging to the solar system, including our Earth (Courtesy ESA) 

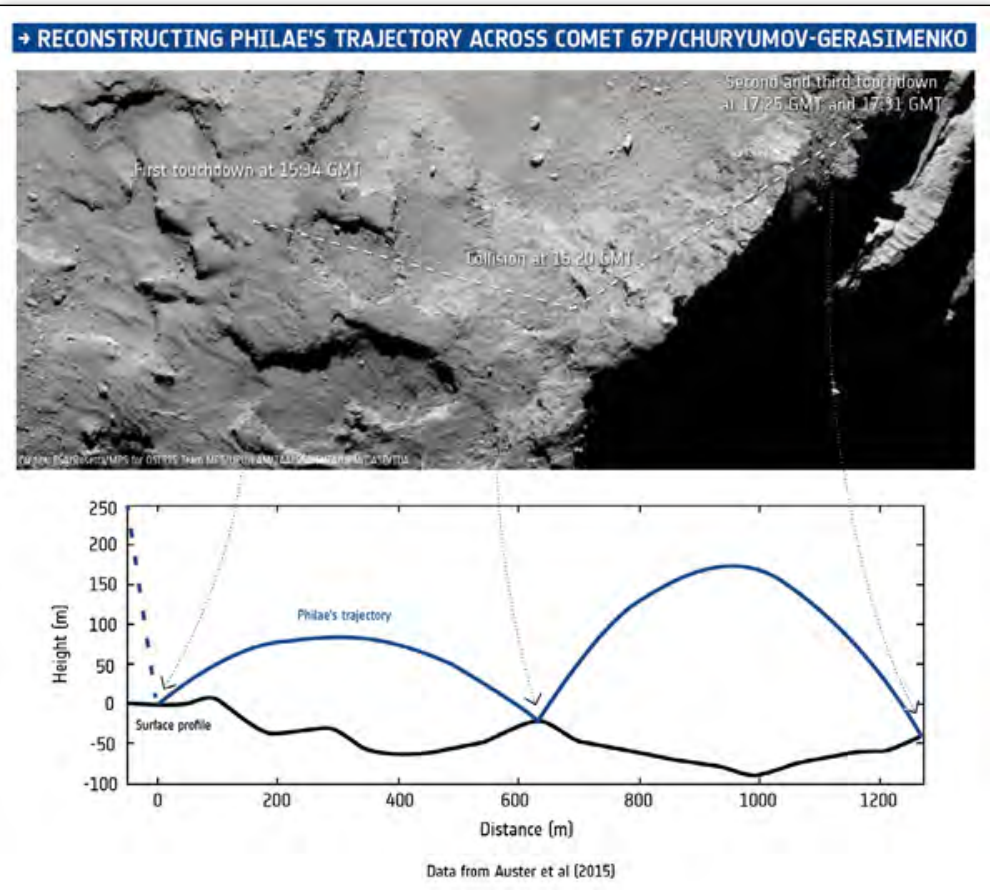

$\triangle \mathrm{FIG}$. 4: A reconstruction of the trajectory by the lander

'Philae' after

first contact with the comet (Courtesy ESA) opaque minerals associated with nonvolatile macromolecular materials (carbon-hydrogen and/or oxygen-hydrogen groups). The coma as monitored by a total gas pressure gage and two mass spectrometers shows substantial time variability and heterogeneity in total gas production and composition, both diurnal and seasonal. The main constituents are $\mathrm{H}_{2} \mathrm{O}$, $\mathrm{CO}$ and $\mathrm{CO}_{2}$ in variable proportions, but a large zoo of molecules, containing various combinations of $\mathrm{H}, \mathrm{C}, \mathrm{O}, \mathrm{N}, \mathrm{S}$ and $\mathrm{P}$ atoms (as they were partially already known from the Giotto mission) have been identified. For the first time $\mathrm{N}_{2}$ could be measured and as the first of the noble gases Ar was clearly identified. The $\mathrm{D} / \mathrm{H}$ ratio in water was found to be three times as high as in terrestrial sea-water (Fig. 3). The comet's water production, at this time of the mission, is not yet fully developed, and the coma may not yet be representative for the inner of the nucleus. Hence firm conclusions still have to wait for the next phase of the mission. Nevertheless the clear identification and relative abundances of the very volatile $\mathrm{N}_{2}$ and Ar strongly suggest that cometary grains formed at very low temperatures (below $30 \mathrm{~K}$ ), and the $\mathrm{D} / \mathrm{H}$ ratio together with the relatively high abundance of Ar tell us that comets of the type of Churyumov-Gerasimenko were not the main providers of the Earth water and volatiles.

\section{Surface science by Philae}

After Philae had come to rest, the attempt to take material out of the nucleus with the drill and hammering of MUPUS into the surface failed, most probably due to the unexpected hardness of the upper surface. As predicted, Philae ceased operations after about $60 \mathrm{hrs}$, when the primary battery was depleted. Analysis of the data, taken during this period, will soon appear in a second Special Issue of Science. The unexpected flight provided a unique opportunity for a joint measurement of the magnetometers on the orbiter and the one on the Lander. Not only could they determine that the nucleus was not magnetized [6], but the measurements helped to reconstruct Philae's trajectory across the surface, including the precise timing of the touchdown points (Fig. 4).

Already this first part of the mission has demonstrated the potential of the payload to achieve its ambitious goals. Its continuation while the comet fully develops its coma promises substantial progress in understanding these messengers from the Solar System's genesis.

\section{About the Authors}

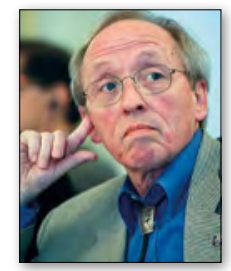

Hans Balsiger was Professor of Physics at the University of Bern (1984-2003). He was PI and CoI on several space missions of ESA and NASA, in particular PI on Giotto to Comet Halley and on Rosetta to Comet Churyumov-Gerasimenko. $\mathrm{He}$ chaired the Solar System Working Group and the Space Science Advisory Committee of ESA and was chairman of ESA's Space Science Program Committee SPC (1996-1999). $\mathrm{He}$ is member of the Academia Europea and of the International Academy of Astronautics IAA, from which he received the Basic Science Award 2004. For his contribution to Apollo 11 he received the NASA Apollo Achievement Award.

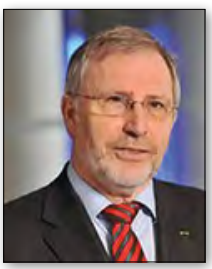

Gerhard Schwehm has been the Rosetta Study and Project Scientist for the mission since the start of the mission studies until 2006, in addition he led Rosetta as Mission Manager from 2004 until 2013. He has also been Deputy Project Scientist for the Giotto mission (1985-1988) and subsequently Project Scientist for the Giotto Extended Mission that passed by comet Grigg-Skjellerup in July 1992.

He headed the Planetary Missions Division in ESA's Science Directorate (2002-2007) in ESTEC, Noordwijk, and spent four years at ESAC, Madrid, as Head of the Solar System Science Operations Division (2007-2011). He retired from ESA in March 2014 but remained involved in the Rosetta Mission as an Advisor.

He has been a member of the Advisory Board of the Ernst-Mach-Institute of the Fraunhofer Society and is a Member of the International Academy of Astronautics and its "Basic Science Commission".

\section{References}

[1] Space Science Horizon 2000, ESA SP 1070

[2] S. S. Genuth, in Popular Culture and the Birth of Modern Cosmology: Comets (Princeton University Press, 1997), 365 pp.

[3] H. Balsiger et al., Nature 321, 330 (1986)

[4] K.-H. Glassmeier et al., Sp. Sci. Rev. 128, 1 (2007)

[5] Science Special Issue 347, (January 2015)

[6] H.-U. Auster et al., Science Express, 14 April 2015 